David's experiments which would enable them to discriminate at all between the possible alternative explanations. Therefore whilst Prof. David had preferred the particular interpretation put forward in his paper, he would have been equally justified by his facts in adopting (had he so chosen) another which some might regard as equally or even more probable than his own". In reply Prof. David rejected my proffered alternative, because (he said) " the rate of partitioning of energy at the high temperature of explosion must be exceedingly rapid, and thermal equilibrium would set in almost instantaneously ".

The alternative view, which I have long held, was further elaborated in the following paragraph on $p$. 202 of the book on "Flame and Combustion in Gases", by Dr. D. T. A. Townend and myself in 1927 : "When an explosive gaseous medium in equilibrium with its environment is ignited, a very sudden and violent change occurs, whereby new molecules are born at a high temperature in an intensely vibrational condition. It is probable that, in the first instance and momentarily, the energy of the newly-born molecules is mainly internal; and that a certain time interval must elapse, during which a rapid process of adjustment between the internal and kinetic energies occurs, before the medium re-attains a steady state of equilibrium with its environment. Indeed, it may be said that, in general, if $\mathrm{T}$ be the maximum mean temperature of the medium on explosion, in all probability its 'energy distribution state' is momentarily different from that which would result had the products of combustion merely been heated up to that temperature. In other words, it is generally agreed that the chemical changes occurring in flames and explosions do generate molecular systems whose energy is, in the first instance, largely vibrational; and that a definite, though it may be small, time interval must elapse before the energy becomes mainly kinetic, and the system re-attains equilibrium with the environment".

In supposing the formation of 'long lived' molecules of carbon dioxide and steam of 'abnormal structure', Prof. David and Mr. Davies have extended the foregoing view much further than I would be prepared to go, especially as regards the "long life" of the supposed metastable molecules. Also, in saying that luminosity was manifested in their exploded gases " at a time long after that at which combination (as inferred from the chemical analysis of rapidly cooled samples) has been completed ", they would seem to have modified the former view about the so-called 'after burning', in the sense that it seems now to be ascribed to incompleteness of the energy development (because of that held up in the supposed metastable molecules) rather than to that of the actual oxidation itself at the moment of maximum pressure.

While reserving any further comments on the matter until after I have studied the evidence adduced in their forthcoming paper, perhaps I may be allowed to put in a caveat that in 'closed-vessel' explosions the relative times for complete inflammation, for the attainment of maximum pressure, and for the cessation of luminosity in the medium, so largely depend upon such factors as shape and size of the enclosure and the occurrence of compression waves, that due caution should be exercised in judging the significance of particular experimental data, especially where the vessels used are larger than would allow of substantial homogeneity throughout the medium at the moment of maximum pressure.

Wilisiam A. Bone.

Imperial College of Science and Technology, London, Feb. 10.

\section{Dissociation Energy of Oxygen Determined from the} Pre-Dissociation of Sulphur Dioxide.

THE absorption spectrum of sulphur dioxide vapour, which we are studying with Wieland, is composed of three electronic regions: the first 3900-3400 which appears at high pressure (1 atmosphere and more in a tube of $100 \mathrm{~cm}$.) ; the second $3370-2450$, which begins to appear at $p=0.1 \mathrm{~mm}$., and the third from 2350 extending beyond 2000 for low pressures $0.02-10 \mathrm{~mm}$.

The first and third regions are made up of series of bands with a fine rotation structure. The second region is also made up of bands with fine structure, but between 2550 and 2500 the bands become broad and diffuse. The molecule is then pre-dissociated.

If we assume that this pre-dissociation limit corresponds to the reaction :

$$
\mathrm{SO}_{2} \rightarrow \mathrm{SO}+\mathrm{O}
$$

the heat of this reaction must be between 111,000 (2550) and $114,000(2500)$ calories, the mean value being 112,500 cal.

We have studied with F. Wolff (Jour. Phys. et Rad., March 1929, p. 81) the spectrum of SO, and have calculated from the convergence limit of the normal term the heat of normal dissociation, $\mathrm{SO} \rightarrow \mathrm{S}+\mathrm{O}-$ 148,000 eal.

From the sharp limit of pre-dissociation at 2792 which we obtained in the absorption spectrum of $\mathrm{S}_{2}$ vapour, we can calculate the heat of dissociation of sulphur : $\mathrm{S}_{2} \rightarrow \mathrm{S}+\mathrm{S}-102,200$ cal. This value is in agreement with the thermal determination 103,600 (Budde, Zeit. an. Chem. 78, 169; 1912). The heat of reaction $\frac{1}{2} \mathrm{~S}_{2}+\mathrm{O}_{2} \rightarrow \mathrm{SO}_{2}$ is 83,000 cal. (Ferguson).

The heat of dissociation of oxygen is, therefore,

$$
\begin{aligned}
148,000+112,000-\frac{102,200}{2}-83,000= \\
126,400 \text { cal. }(5 \cdot 5 \text { volt }) .
\end{aligned}
$$

This value is in good agreement with the determination which we made from the pre-dissociation of $\mathrm{NO}_{2}$ (128,000 cal.) (NATURE, Feb. 8, p. 202).

It is very probable that the third absorption region of $\mathrm{SO}_{2}$ presents also a pre-dissociation limit corresponding to the reaction :

$$
\mathrm{SO}_{2} \rightarrow \mathrm{SO}+\mathrm{O}^{*}
$$

We have found that the energy level of the metastable $1 D$ oxygen is equal to $13,700 \mathrm{~cm}^{-1}$ (NATURE, Feb. 8). The second pre-dissociation must then occur between 1800 and $1900 \mathrm{~A}$. We are studying it now with the vacuum spectrograph.

\section{Institute of Physical Chemistry,} Zurich.

\section{Non-Disjunction Produced by Carbon Dioxide.}

THE abnormal mitoses seen in tissue cultures kept under high tensions of carbon dioxide were described in a communication in NATURE of Mar. 17, 1928, p. 420 (see also Brit. J. Exp. Path., 9, 240; 1928). Fragmentation of the chromosomes and irregular migration of the chromatin to the controsomes were seen, exactly similar to the mitotic changes observed many years previously in the eggs of Ascaris megalocephala subjected to radium (Arch. Middx. Hosp., 30, 98; 1913). These observations suggested that carbon dioxide might produce hereditary disturbances, when applied to germ cells, analogous to those produced by $\mathrm{X}$-rays and radium. In order to test this, observations on non-disjunction in Drosophila have been made, in which newly hatched virgin red-eyed flies were subjected to either pure carbon dioxide for a few hours,

No. 3147, VoL. 125] 